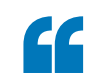

multiple

components of the pathogenic axis are

suitable for the development of novel drug treatments for SRNS
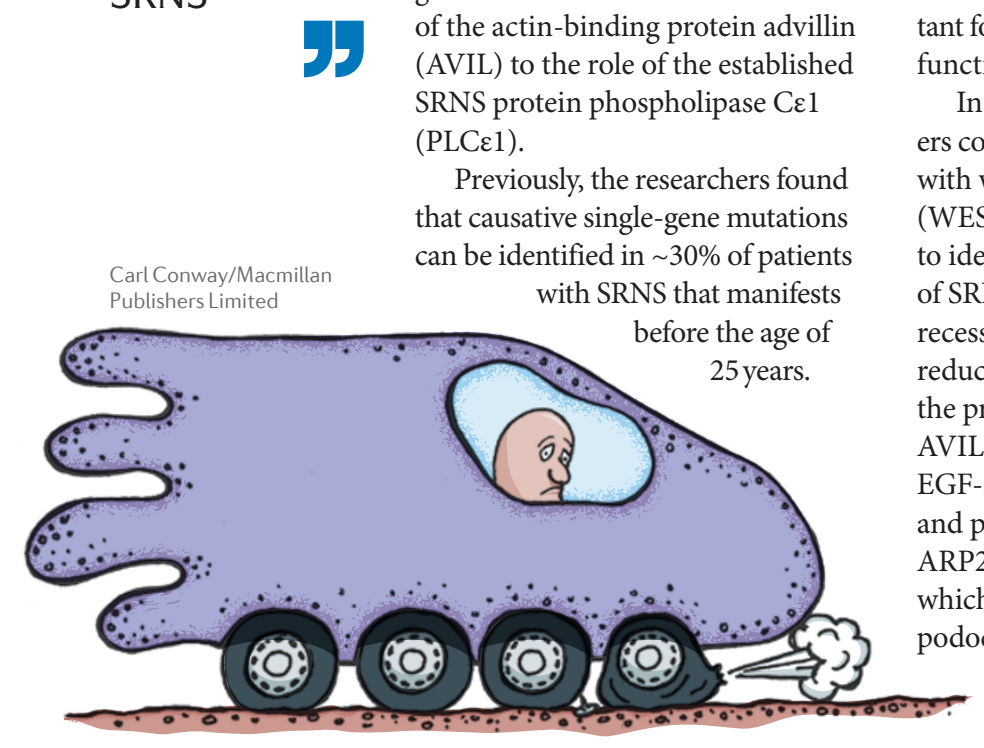

NEPHROTIC SYNDROME

The disease mechanisms of steroidresistant nephrotic syndrome (SRNS) are poorly understood. Now, Shazia Ashraf, Friedhelm Hildebrandt and colleagues delineate a novel pathogenic axis that links loss of function of the actin-binding protein advillin (AVIL) to the role of the established SRNS protein phospholipase $\mathrm{C} \varepsilon 1$ (PLCE1).

Previously, the researchers found that causative single-gene mutations can be identified in $\sim 30 \%$ of patients with SRNS that manifests

\title{
AVIL mutations reduce podocyte migration rate in SRNS
}

"Most of the encoded gene products are expressed in podocytes, confirming that loss of podocyte function has a critical role in the pathogenesis of SRNS, and that these proteins and their functional pathways are important for the maintenance of glomerular function," comments Ashraf.

In their recent study, the researchers combined homozygosity mapping with whole-exome sequencing (WES) in consanguineous families to identify novel monogenic causes of SRNS. They discovered three ecessive mutations in AVIL that duced the actin-bundling ability of he protein in vitro. "Knockdown of VIL in human podocytes blocked GF-induced PLC 1 signalling and prevented assembly of the RP2/3 complex in lamellipodia, hich resulted in an attenuated docyte migration rate (PMR)," says Ashraf. The PMR could be rescued by overexpression of wild-type AVIL or PLCe1, but not by overexpression of the patient-derived AVIL mutants. Furthermore, an increase in PMR owing to overexpression of wild-type AVIL, PLCe1 or EGF stimulation was abrogated by inhibition of the ARP2/3 complex, indicating that ARP2/3-dependent lamellipodia formation is downstream of AVIL and PLCE1 function.

The researchers suggest that multiple components of the pathogenic axis are suitable for the development of novel drug treatments for SRNS. They also propose that WES should be offered to every patient who has persistent proteinuria occurring before the age of 25 years. "WES will provide the patient with an unequivocal diagnosis, might uncover a form of nephrotic syndrome that is amenable to treatment and could avoid the need for renal biopsies, as well as further unravel the mechanisms of pathogenic pathways and enable the generation of functional assays for drug discovery," says Ashraf. Jack M. Heintze

ORIGINAL ARTICLE Rao, J. et al. Advillin acts upstream of phospholipase $C \varepsilon 1$ in steroidresistant nephrotic syndrome. J. Clin. Invest. http://dx.doi.org/10.1172/JCl94138 (2017) 